\title{
Transmission of Nonuniform Memoryless Sources via Nonsystematic Turbo Codes
}

\author{
Guang-Chong Zhu, Member, IEEE, Fady Alajaji, Senior Member, IEEE, Jan Bajcsy, Member, IEEE, and \\ Patrick Mitran, Student Member, IEEE
}

\begin{abstract}
We investigate the joint source-channel coding problem of transmitting nonuniform memoryless sources over binary phase-shift keying-modulated additive white Gaussian noise and Rayleigh fading channels via turbo codes. In contrast to previous work, recursive nonsystematic convolutional encoders are proposed as the constituent encoders for heavily biased sources. We prove that under certain conditions, and when the length of the input source sequence tends to infinity, the encoder state distribution and the marginal output distribution of each constituent recursive convolutional encoder become asymptotically uniform, regardless of the degree of source nonuniformity. We also give a conjecture (which is empirically validated) on the condition for the higher order distribution of the encoder output to be asymptotically uniform, irrespective of the source distribution. Consequently, these conditions serve as design criteria for the choice of good encoder structures. As a result, the outputs of our selected nonsystematic turbo codes are suitably matched to the channel input, since a uniformly distributed input maximizes the channel mutual information, and hence, achieves capacity. Simulation results show substantial gains by the nonsystematic codes over previously designed systematic turbo codes; furthermore, their performance is within 0.74-1.17 $\mathrm{dB}$ from the Shannon limit. Finally, we compare our joint source-channel coding system with two tandem schemes which employ a fourth-order Huffman code (performing near-optimal data compression) and a turbo code that either gives excellent waterfall bit-error rate (BER) performance or good error-floor performance. At the same overall transmission rate, our system offers robust and superior performance at low BERs $\left(\leq 10^{-4}\right)$, while its complexity is lower.
\end{abstract}

Index Terms-Additive white Gaussian noise (AWGN) and Rayleigh fading channels, joint source-channel coding, nonsystematic turbo codes, nonuniform independent and identically distributed (i.i.d.) sources, Shannon limit.

Paper approved by A. K. Khandani, the Editor for Coding and Information Theory of the IEEE Communications Society. Manuscript received December 17, 2002; revised September 2, 2003. This work was supported in part by the Natural Sciences and Engineering Research Council (NSERC) of Canada and the Premier's Research Excellence Award (PREA) of Ontario. This paper was presented in part at the Conference on Information Sciences and Systems, Princeton, NJ, March 2002, and in part at the 2nd IASTED International Conference on Wireless and Optical Communications, Banff, AB, Canada, July 2002.

G.-C. Zhu was with the Department of Mathematics and Statistics, Queen's University, Kingston, ON K7L 3N6, Canada. He is now with the Department of Electrical and Computer Engineering, University of Toronto, Toronto, ON M5S 3G4, Canada.

F. Alajaji is with the Department of Mathematics and Statistics, Queen's University, Kingston, ON K7L 3N6, Canada (e-mail: fady@ mast.queensu.ca).

J. Bajcsy is with the Department of Electrical and Computer Engineering, McGill University, Montreal, QC H3A 2A7, Canada.

P. Mitran is with the Division of Engineering and Applied Sciences, Harvard University, Cambridge, MA 02138 USA.

Digital Object Identifier 10.1109/TCOMM.2004.833026

\section{INTRODUCTION}

$\mathbf{I}$ $\mathrm{N}$ MOST OF the theory and practice of error-control coding, the input to the channel encoder is assumed to be uniform, independent, and identically distributed (i.i.d.); i.e., the source generates a memoryless binary stream $\left\{U_{k}\right\}_{k=0}^{\infty}$, where $\operatorname{Pr}\left\{U_{k}=0\right\}=1 / 2$. In reality, however, a substantial amount of redundancy resides in natural sources. For example, many uncompressed binary images (e.g., facsimile and medical images) may contain as much as $80 \%$ of redundancy in the form of nonuniformity (e.g., [1], [2]); this corresponds to a probability $p_{0} \triangleq \operatorname{Pr}\left\{U_{k}=0\right\}=0.97$. In this case, a source encoder would be used. Such an encoder is optimal, if it can eliminate all the source redundancy and generates uniform i.i.d. outputs. However, most existing source encoders are suboptimal, particularly fixed-length encoders that are commonly used for transmission over noisy channels; thus, the source-encoder output contains some residual redundancy. For example, the $4.8 \mathrm{~kb} / \mathrm{s}$ US FS 1016 CELP speech vocoder produces line spectral parameters that contain $41.5 \%$ of residual redundancy due to nonuniformity and memory [3]. Variable-length or entropy codes (e.g., Huffman codes), which can be asymptotically optimal (for sufficiently large blocks of source symbols), could be employed instead of fixed-length codes or in conjunction with them. However, error-propagation problems in the presence of channel noise are inevitable, and are sometimes catastrophic. Therefore, the reliable communication of sources with a considerable amount of redundancy (residual if compressed, or natural if not) is an important issue. This, in essence, is a joint source-channel coding problem. Blizard [4], Koshelev [5], and Hellman [6] are among the first few who proposed convolutional coding for the joint source-channel coding of sources with natural redundancy, where the source statistics are used at the receiver. Specifically, the convolutional encoding of such sources (Markov and nonuniform sources) over memoryless channels, and their decoding via sequential decoders employing a decoding metric that is dependent on both source and channel distributions, were studied in [4] and [5]. The computational complexity of such sequential decoders is analyzed, and it is shown that for a range of transmission rates, the expected number of computations per decoded bit is finite. In [6], a lossless joint source-channel coding theorem for convolutional codes is established. It is proved that for a discrete memoryless source and channel pair, there exist convolutional codes of rate $R$ that can be used to perform reliable joint source and channel coding (under maximum a posteriori (MAP) decoding) as long as $R<C / H$, where $H$ is the source entropy and $C$ is the channel capacity. Recently, several studies 
(e.g., [1]-[3], [7]-[22], etc.) have also shown that appropriate use of the source redundancy can significantly improve the system performance.

Turbo codes have been regarded as one of the most exciting breakthroughs in channel coding. The original work by Berrou et al. demonstrated excellent performance of turbo codes for uniform i.i.d. sources over additive white Gaussian noise (AWGN) channels [23]. The work was later extended to Rayleigh fading channels showing comparable performance [24]. Recently, McEliece pointed out that turbo codes have also great potential on nonstandard channels (asymmetric, nonbinary, multiuser, etc.) [25]. However, the above papers focus on uniform i.i.d. sources. In [26] and [27], the authors considered using turbo codes for sources with memory. To the best of our knowledge, the issue of designing turbo codes for nonuniform i.i.d. sources has not been fully studied, except for the recent work in [28] and [29], in which standard systematic turbo codes (STCs) are considered, where each constituent encoder is a recursive systematic convolutional (RSC) encoder. Although the gains achieved by these codes are considerable with respect to the original Berrou code, their performance gaps vis-a-vis the Shannon limit, also known as the optimal performance theoretically achievable (OPTA), are still relatively big for heavily biased sources (e.g., with $p_{0}=0.8,0.9$ ). Analysis on the encoder output reveals that the drawback lies in the systematic structure, which results in a mismatch ${ }^{1}$ between the biased distribution of the systematic bit stream and the uniform input distribution needed to achieve channel capacity. As we will show in this paper, when some constraints are satisfied, recursive nonsystematic convolutional (RNSC) encoders can generate asymptotically uniform outputs, even for extremely biased sources. But it is known that the capacity of a binary-input AWGN or Rayleigh channel is achieved by a uniform i.i.d. channel input. Furthermore, it was shown in [30] by Shamai and Verdú that the empirical distribution of any good code (a code with rate close to capacity and vanishing probability of error for sufficiently long blocklengths) should approach the capacity-achieving input distribution. ${ }^{2}$ Therefore, we propose using RNSC turbo encoders. Simulation results demonstrate substantial gains over STCs. The OPTA gaps for heavily biased sources are, hence, significantly reduced.

This paper is organized as follows. In Section II, we illustrate the need to examine nonsystematic turbo codes (NSTCs) instead of systematic codes for the transmission of strongly nonuniform sources by evaluating the capacity loss incurred when such sources are directly (e.g., via a systematic bit stream) sent over binary phase-shift keying (BPSK)-modulated AWGN or Rayleigh fading channels. In Section III, we give two asymptotic properties (when the input sequence length

\footnotetext{
${ }^{1} \mathrm{~A}$ similar mismatch in the context of the design of scalar quantizers for nonuniform memoryless sources over binary symmetric channels (BSC) was also observed in [15] and [17], and addressed via the use of a rate-one convolutional encoder. This method is, however, unsuitable for our problem, as it will result in error propagation at the receiver due to our use of large data blocks in an attempt to achieve the Shannon limit.

${ }^{2}$ More precisely, it is shown in [30, Th. 4] that for any discrete memoryless channel, and for any fixed integer $k>0$, the $k$ th-order empirical distribution of any regular good code sequence converges (in the Kullback-Leibler divergence sense) to the $k$-product of the capacity-achieving distribution.
}

tends to infinity) of recursive convolutional encoders whose input is a nonuniform i.i.d. source with arbitrary degree of nonuniformity. Design criteria for good nonsystematic encoder structures based on these two properties are introduced in Section IV. The iterative decoding design for such NSTCs is also addressed. Simulation results and performance comparisons to the Shannon limit are presented in Section V. In Section VI, we compare our joint source-channel coding system with two tandem coding schemes that employ a nearly optimal source code followed by Berrou's (37,21) turbo code [23] (which gives excellent waterfall bit-error rate (BER) performance), or a systematic $(35,23)$ turbo code (which gives a slightly inferior waterfall BER performance but a lower error-floor performance). Finally, conclusions are given in Section VII.

\section{CAPACITy Loss DUE TO CHANNEL-INPUT MismatCH}

In this section, we illustrate why systematic codes are not well matched to symmetric channels (due to their systematic bit stream) when the source is very biased $\left(p_{0}=0.8,0.9\right)$. This is achieved by examining the capacity loss incurred in BPSKmodulated AWGN channels and memoryless Rayleigh fading channels with known channel state information (CSI) [24] under such biased sources as input. The channel capacity is the largest rate at which information can be transmitted (via a block code) and recovered with a vanishingly low probability of error. For discrete-input memoryless channels, it is well known that the capacity is given by the maximum of the mutual information between the channel input and output: $C=\max _{p(x)} I(X ; Y)$, where the maximization is taken with respect to all input distributions $p(x)$. When the channel is symmetric, the capacity is achieved by a uniform channel input distribution. For AWGN channels, the capacity is

$$
C_{\mathrm{AWGN}}=1-\int_{-\infty}^{\infty} \frac{1}{\sqrt{2 \pi \sigma^{2}}} e^{-(y+1)^{2} / 2 \sigma^{2}} \log _{2}\left(1+e^{2 y / \sigma^{2}}\right) d y .
$$

Similarly, for Rayleigh fading channels, the capacity is

$$
\begin{aligned}
C_{\text {Ray }}= & 1-\int_{0}^{\infty} \int_{-\infty}^{\infty} \frac{1}{\sqrt{2 \pi \sigma^{2}}} e^{-(y+a)^{2} / 2 \sigma^{2}} \\
& \cdot 2 a e^{-a^{2}} \cdot \log _{2}\left(1+e^{2 a y / \sigma^{2}}\right) d y d a .
\end{aligned}
$$

Note that the average energy per channel symbol $E_{s}=1$, and $\sigma^{2}=N_{0} / 2$ is the variance of the AWGN. Therefore, both $C_{\mathrm{AWGN}}$ and $C_{\text {Ray }}$ are functions of the signal-to-noise ratio (SNR) $E_{s} / N_{0}$

When a nonuniform i.i.d. source (with distribution $p_{0} \neq 1 / 2$ ) is directly fed into either channel, the channel input is obviously nonuniform, and the capacity cannot be fully exploited since the uniform distribution achieves capacity for such channels. Therefore, the actual achievable capacity for AWGN channels with such a biased input is

$$
\begin{aligned}
& C_{\mathrm{AWGN}}^{\mathrm{bias}} \\
& =\left.I(X ; Y)\right|_{P(X=0)=p_{0}} \\
& =-p_{0} \int_{-\infty}^{\infty} \frac{1}{\sqrt{2 \pi \sigma^{2}}} e^{-(y+1)^{2} / 2 \sigma^{2}} \log _{2}\left(p_{0}+p_{1} e^{2 y / \sigma^{2}}\right) d y \\
& \quad-p_{1} \int_{-\infty}^{\infty} \frac{1}{\sqrt{2 \pi \sigma^{2}}} e^{-(y-1)^{2} / 2 \sigma^{2}} \log _{2}\left(p_{0} e^{-2 y / \sigma^{2}}+p_{1}\right) d y
\end{aligned}
$$


TABLE I

ILLUSTRATION OF CHANNEL-INPUT MISMATCH FOR AWGN AND RAYLEIGH FADING CHANNELS: $C$ versus $C^{\text {bias }}$ FOR $p_{0}=0.8,0.9$

\begin{tabular}{c||c|c||c|c||c|c}
\hline \hline \multirow{3}{*}{ Channel } & \multicolumn{1}{|c||}{$\begin{array}{c}\text { SNR }(\mathrm{dB}) \\
E_{s} / N_{0}\end{array}$} & \multicolumn{1}{c||}{$C$} & \multicolumn{2}{c||}{$p_{0}=0.8$} & \multicolumn{2}{c}{$p_{0}=0.9$} \\
\cline { 3 - 7 } & $C^{\text {bias }}$ & $\frac{C^{\text {bias }}}{C}$ & $C^{\text {bias }}$ & $\frac{C^{\text {bias }}}{C}$ \\
\hline \hline \multirow{4}{*}{ AWGN } & -4.0 & 0.415 & 0.284 & $68.5 \%$ & 0.172 & $41.4 \%$ \\
\cline { 2 - 7 } & -2.0 & 0.564 & 0.392 & $69.6 \%$ & 0.242 & $42.9 \%$ \\
\cline { 2 - 7 } & 0.0 & 0.721 & 0.509 & $70.6 \%$ & 0.320 & $44.4 \%$ \\
\cline { 2 - 7 } & 2.0 & 0.860 & 0.614 & $71.4 \%$ & 0.392 & $45.6 \%$ \\
\cline { 2 - 7 } & 4.0 & 0.951 & 0.684 & $71.9 \%$ & 0.442 & $46.4 \%$ \\
\hline \hline \multirow{4}{*}{ Rayleigh } & -4.0 & 0.348 & 0.240 & $69.0 \%$ & 0.147 & $42.2 \%$ \\
\cline { 2 - 7 } & -2.0 & 0.454 & 0.317 & $69.8 \%$ & 0.197 & $43.3 \%$ \\
\cline { 2 - 7 } & 0.0 & 0.566 & 0.398 & $70.4 \%$ & 0.250 & $44.2 \%$ \\
\cline { 2 - 7 } & 2.0 & 0.671 & 0.476 & $71.0 \%$ & 0.302 & $45.0 \%$ \\
\cline { 2 - 7 } & 4.0 & 0.763 & 0.544 & $71.3 \%$ & 0.348 & $45.6 \%$ \\
\hline \hline
\end{tabular}

and for Rayleigh fading channels, it is

$$
\begin{aligned}
C_{\text {Ray }}^{\text {bias }}= & \left.I(X ; Y)\right|_{P(X=0)=p_{0}} \\
= & -p_{0} \int_{0}^{\infty} \int_{-\infty}^{\infty} \frac{1}{\sqrt{2 \pi \sigma^{2}}} e^{-(y+a)^{2} / 2 \sigma^{2}} \cdot 2 a e^{-a^{2}} \\
& \cdot \log _{2}\left[p_{0}+p_{1} e^{2 a y / \sigma^{2}}\right] d y d a \\
- & p_{1} \int_{0}^{\infty} \int_{-\infty}^{\infty} \frac{1}{\sqrt{2 \pi \sigma^{2}}} e^{-(y-a)^{2} / 2 \sigma^{2}} \cdot 2 a e^{-a^{2}} \\
& \cdot \log _{2}\left[p_{0} e^{-2 a y / \sigma^{2}}+p_{1}\right] d y d a
\end{aligned}
$$

As a result, if one transmits the nonuniform source directly, the capacity cannot be fully realized, due to the mismatch between the channel input distribution and the capacity-achieving distribution. This is numerically illustrated in Table I for various values of SNR, where we note that in certain cases, $C^{\text {bias }} / C$ can be as low as $41.4 \%$. This leads us to conclude that for the transmission of very biased nonuniform sources, we should eliminate the systematic bit stream in the encoder structure, and hence, investigate the design of nonsystematic codes in our attempt to avoid such mismatch-incurred performance loss.

\section{ASYMPTOTIC PROPERTIES OF RECURSIVE ENCODERS}

In this section, we prove two asymptotic properties of recursive convolutional encoders when the input sequence is a nonuniform i.i.d. source $\left\{U_{k}\right\}$, with distribution $p_{0}$ and length approaching infinity. Throughout this section, we assume that $p_{0} \in(0.0,1.0)$ and that $p_{0} \neq 1 / 2$. We first show that, regardless of the value of $p_{0}$, when certain conditions are satisfied, the parity output of the encoder is asymptotically uniform. Based upon empirical observations, we also give a conjecture on the condition when the encoder output has higher order asymptotically uniform distributions (the joint distribution of $m>1$ consecutive parity bits). We next show that, if the feedback tap coefficient of the last memory element is unity, all of the encoder states will be reached with asymptotically equal probability. The conditions for the above two asymptotic properties are both necessary and sufficient. These two properties offer pertinent design criteria, which will be used in the next section for constructing good encoder structures of turbo codes for heavily biased, nonuniform i.i.d. sources.

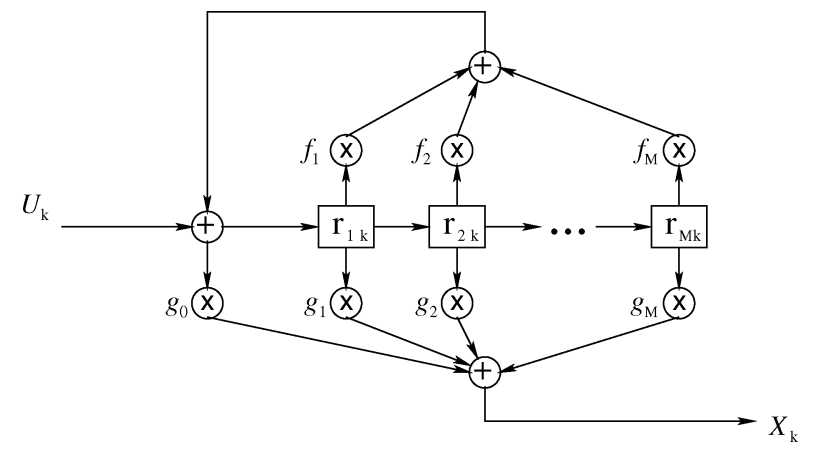

Fig. 1. General structure of a recursive convolutional encoder.

We first study the asymptotic distribution of a single paritycheck output of a constituent recursive convolutional encoder (either systematic or nonsystematic, see Fig. 1). We begin by quoting the following result from [33, Th., p. 1692] with $Q=2$ and [34, Lemma 3, p. 1855].

Lemma 1: Consider the sequence $\left\{Y_{n} z_{n}\right\}$, where $\left\{Y_{n}\right\}$ is a stationary sequence of independent symbols from $\mathrm{GF}(2)$ with probability $P\left(Y_{n}=0\right)=p_{0}$, and $\left\{z_{n}\right\}$ is a deterministic sequence containing an infinite number of nonzero terms. If $W_{k}=\sum_{n=0}^{k} Y_{n} z_{n}$, where the summation is modulo-2, then

$$
\lim _{k \rightarrow \infty} P\left(W_{k}=0\right)=\frac{1}{2} .
$$

Using the above lemma, we can now establish the following result.

Theorem 1: For a recursive convolutional encoder with feedback polynomial $F(D)$ and feed-forward polynomial $G(D)$, the necessary and sufficient condition that the encoder output is asymptotically uniform for a nonuniform i.i.d. source with distribution $p_{0}$ (regardless of the value of $p_{0}$ ) is that $G(D)$ is not divisible by $F(D)$ in $\mathrm{GF}(2)$.

Proof:

Necessary Part: Suppose the source generates $\left\{U_{k}\right\}_{k=0}^{\infty}$, and the encoder produces an output sequence of $\left\{X_{k}\right\}_{k=0}^{\infty}$. Denote the encoder input and output in polynomial forms as $U(D)=\sum_{k=0}^{\infty} U_{k} D^{k}$ and $X(D)=\sum_{k=0}^{\infty} X_{k} D^{k}$, respectively. Also, define $E(D) \triangleq G(D) / F(D)$. Then for a recursive convolutional encoder with feedback polynomial $F(D)$ and feed-forward polynomial $G(D)$, we have

$$
X(D)=U(D) \cdot \frac{G(D)}{F(D)} \triangleq U(D) \cdot E(D) .
$$

Suppose that $G(D)$ is divisible by $F(D)$ in $\mathrm{GF}(2)$. Then $E(D)$ is a polynomial of $D$ with finite degree. Therefore, any bit of the encoder output $X_{k}$ is essentially a modulo-2 summation of a finite number of bits from $\left\{U_{k}\right\}_{k=0}^{\infty}$. However, a finite modulo-2 summation of nonuniform i.i.d. variables would still be nonuniform; therefore, the encoder output $\left\{X_{k}\right\}_{k=0}^{\infty}$ is nonuniform.

Sufficient Part: When $G(D)$ is not divisible by $F(D)$ in $\mathrm{GF}(2), E(D)$ is an infinite polynomial of $D$

$$
E(D)=e_{0}+e_{1} D+e_{2} D^{2}+\cdots+e_{k} D^{k}+\cdots
$$


where $e_{k}$ is binary. Then

$$
X_{k}=\sum_{l=0}^{k} U_{l} e_{k-l}, \quad k=0,1,2, \ldots
$$

(where summation is in mod-2).

Letting $z_{l} \triangleq e_{k-l}$, where $l=0,1, \ldots, k$. Since $E(D)=$ $G(D) / F(D)$ is a rational polynomial fraction, when $G(D)$ is not divisible by $F(D)$ in $\mathrm{GF}(2),\left\{e_{l}\right\}$ is a periodic series. Therefore, when $k \rightarrow \infty$, the number of ones in $\left\{e_{l}\right\}_{l=0}^{k}$ (and hence, in $\left\{z_{l}\right\}_{l=0}^{k}$ ) also goes to infinity. From Lemma 1 , we obtain that $\lim _{k \rightarrow \infty} P\left(X_{k}=0\right)=1 / 2$.

In [30], Shamai and Verdú proved that for any fixed positive integer $k$, the $k$ th-order empirical distribution of any good code (i.e., a code approaching capacity with asymptotically vanishing probability of error) converges to the input distributions that achieve channel capacity. The capacity of a binary-input AWGN or Rayleigh channel is achieved when its mutual information is maximized by an i.i.d. uniform input. Therefore, having higher order uniform distributions (in addition to the first-order distribution) in encoder outputs is also desirable. A related result has been established by Leeper in [34], which states that for any binary source (with unknown statistics), recursive convolutional encoders can produce a parity output whose firstand second-order distributions can be arbitrarily close (within a given value $\delta$ ) to uniform for an appropriately chosen large value of the source sequence under the following conditions: 1 ) the source is first passed through the equivalent of a BSC with an arbitrarily small, but nonzero, error probability $\epsilon ; 2$ ) the encoder's memory size is greater than a certain value determined by $\delta$ and $\epsilon$. For our system, which does not satisfy the above (rather stringent) conditions, we can state the following conjecture based on extensive simulations.

Conjecture: Suppose a nonuniform i.i.d. source with distribution $p_{0}$ is input to a recursive convolutional encoder with feedback polynomial $F(D)$ and feed-forward polynomial $G(D)$. If $G(D) / F(D)$ is in its minimal form, where $M$ is the degree of $F(D)$, then the $m$ th-order distribution of the encoder output is asymptotically uniform for any $p_{0}$ and $\forall m=1,2, \ldots, M$.

A proof for the above conjecture with arbitrary $G(D)$ and $F(D)$ is not obvious. However, the conjecture has been verified by empirical estimations of all possible combinations of recursive convolutional encoders with memory 4 .

The above results indicate promising potential when RNSC encoders are used as the constituent turbo-code encoders for the transmission of nonuniform i.i.d. sources. However, in this large family of candidate encoders, some structures offer inferior performance. Consider a heavily biased nonuniform i.i.d. sequence which is used as the input of a recursive convolutional encoder; for some structures, with high probability the state transition is confined within a few encoder states, while other states may rarely or never be reached. This inefficient use of the encoder memory would result in performance degradations. Therefore, we next establish the condition for the asymptotic uniformity on the distribution of the encoder states. This result will be a useful design criterion in eliminating poor encoder structures.

Lemma 2: For a recursive convolutional encoder, as depicted in Fig. 1, at time $k+1$, each state $s_{k+1}$ can be reached from only two distinct (state, input) pairs $\left(s_{k}^{(0)}, u_{k+1}^{(0)}\right)$ and $\left(s_{k}^{(1)}, u_{k+1}^{(1)}\right)$, where the two states at time $k$ satisfy $s_{k}^{(0)} \neq s_{k}^{(1)}$. The necessary and sufficient condition for $u_{k+1}^{(0)} \neq u_{k+1}^{(1)}$ is $f_{M}=1$.

Proof: The encoder state is determined by the content of each memory element of the shift register. Let the state at time $k$ be $s_{k}=\left(r_{1 k}, r_{2 k}, \ldots, r_{M k}\right)$. By the shift register's structure, we have

$$
\begin{aligned}
& r_{1, k+1}=u_{k+1} \oplus \sum_{j=1}^{M} f_{j} \cdot r_{j k} \\
& r_{j, k+1}=r_{j-1, k}, \quad j=2,3, \ldots, M
\end{aligned}
$$

where $\oplus$ and the summation are modulo- 2 . Therefore, $s_{k+1}$ can only be reached from two states which only differ at $r_{M k}$. Now rewrite (1) as follows:

$$
u_{k+1}=r_{1, k+1} \oplus f_{M} \cdot r_{M k} \oplus \sum_{j=1}^{M-1} f_{j} \cdot r_{j k} .
$$

For both states, $s_{k}^{(0)}$ and $s_{k}^{(1)}$, the summand produces the same result. When $f_{M}=0$, the input $u_{k+1}$ is independent of the encoder state being $s_{k}^{(0)}$ or $s_{k}^{(1)}$. When $f_{M}=1$, different values of $r_{M k}$ require different values of $u_{k+1}$.

Theorem 2: Consider a recursive convolutional encoder with feedback $\left\{f_{1}, f_{2}, \ldots, f_{M}\right\}$, and let a nonuniform i.i.d. source with distribution $p_{0}$ be its input. Then the encoder state distribution is asymptotically uniform (as the source sequence length tends to infinity), regardless of the value of $p_{0}$ iff $f_{M}=1$.

Proof: The shift registers' states form a fully connected (irreducible) Markov chain when $p_{0} \in(0,1)$; so the state distribution will asymptotically converge to the steady-state distribution. Then it is equivalent to show that the uniform distribution is or is not the steady-state distribution. From Lemma 2, there are only two (state, input) pairs $\left(s_{k}^{(0)}, u_{k+1}^{(0)}\right)$ and $\left(s_{k}^{(1)}, u_{k+1}^{(1)}\right)$ that may transit to state $s_{k+1}$. Without loss of generality, denote the possible encoder state as $0,1, \ldots, 2^{M}-1$. Assuming that at time $k$ the state distribution is uniform, i.e., $\operatorname{Pr}\left(S_{k}=s\right)=2^{-M}$ for $s=0,1, \ldots, 2^{M}-1$, then at time $k+1$, if $f_{M}=1$, we have

$$
\begin{aligned}
& \operatorname{Pr}\left(S_{k+1}=s_{k+1}\right) \\
& =\operatorname{Pr}\left(S_{k}=s_{k}^{(0)}, U_{k+1}=u_{k+1}^{(0)}\right)+\operatorname{Pr}\left(S_{k}=s_{k}^{(1)}, U_{k+1}=u_{k+1}^{(1)}\right) \\
& =\operatorname{Pr}\left(U_{k+1}=u_{k+1}^{(0)}\right) 2^{-M}+\operatorname{Pr}\left(U_{k+1}=u_{k+1}^{(1)}\right) 2^{-M}=2^{-M}
\end{aligned}
$$

where the second equality is due to the independence of $S_{k}$ and $U_{k+1}$, and the last equality holds because $u_{k+1}^{(0)} \neq u_{k+1}^{(1)}$ by Lemma 2. Therefore, $f_{M}=1$ yields that the uniform distribution is the steady distribution of the encoder states. On the other hand, if $f_{M}=0$, by Lemma $2, u_{k+1}^{(0)}=u_{k+1}^{(1)}$; then $\operatorname{Pr}\left(S_{k+1}=\right.$ $\left.s_{k+1}\right)=2 \operatorname{Pr}\left(U_{k+1}=u_{k+1}\right) \cdot 2^{-M}$. Thus, when the source is biased, the uniform distribution is not the steady-state distribution when $f_{M}=0$. In other words, when $f_{M}=0$, biased sources result in a biased distribution of the encoder states, and the steady-state distribution is not equal to the uniform distribution. Hence, for any $p_{0} \in(0,1), p_{0} \neq 1 / 2$, the encoder state distribution is asymptotically uniform iff $f_{M}=1$. 


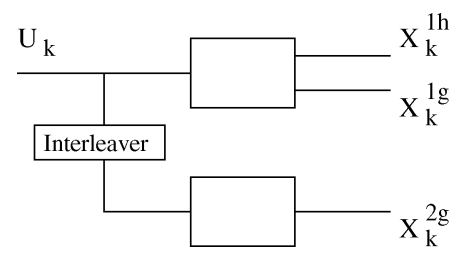

Fig. 2. Nonsystematic turbo-encoder structure a).

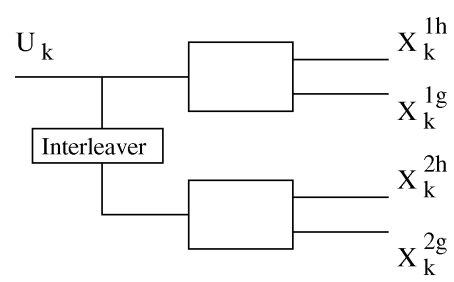

Fig. 3. Nonsystematic turbo-encoder structure b).

\section{NSTCs}

In most of the turbo-codes literature, the turbo-code encoders are systematic; however, NSTCs were recently proposed by Costello et al. [35], followed by a series of papers in [36]-[38]. The scenario in these papers is still for uniform i.i.d. sources. The motivation for using NSTCs as an alternative to their systematic peers is due to their larger code space; therefore, there is a potential that better codes might be found. In [38], Massey et al. construct an asymmetric NSTC that outperforms Berrou's $(37,21)$ code by about $0.2 \mathrm{~dB}$ at the $10^{-5}$ BER level with a block-length size of 4096. For larger block lengths, we expect that Massey's code will still outperform Berrou's code, although it is not clear if the $0.2-\mathrm{dB}$ gain would be maintained.

As illustrated in Section II, when nonuniform i.i.d. sources get heavily biased, a possibly significant performance loss may result, due to the systematic structure. Furthermore, in light of Shamai and Verdú's result [30], Theorem 1, and the conjecture, we note that NSTCs seem to provide a suitable solution for the source-channel coding of such sources.

\section{A. Design of Good Encoder Structures}

Figs. 2 and 3 show our proposed nonsystematic turbo encoders. In a), the first constituent encoder has two parity outputs $X_{k}^{1 h}$ and $X_{k}^{1 g}$, while the second has only one parity output $X_{k}^{2 g}$, so the overall rate is $1 / 3$. This structure has been extensively used in [35]-[38]. In b), both constituent encoders have two parity outputs, and the overall rate is $1 / 4$. Structure b) can achieve the same overall rate of $1 / 3$ via puncturing. It is also clear that structure a) is a special case of structure b), obtained by completely puncturing $X_{k}^{2 h}$; therefore, a generally designed decoder for structure b) can also be employed for structure a).

Ideally, good design criteria are analytically based on the minimization of the error probability. However, to the best of our knowledge, all available error bounds for turbo codes are obtained by averaging over a code ensemble or by assuming uniform interleaving and maximum-likelihood (ML) decoding, while turbo codes in fact employ random interleaving, with each constituent decoder adopting the Bahl-Cocke-Jelinek-Raviv (BCJR) algorithm [39], which is a MAP decoding algorithm.
Furthermore, the bounds are useful only in the error-floor region at high SNRs; they are not tight in the waterfall region [40]-[42]. Since our goal is to obtain the best waterfall performance, we revert to other methods to find good design criteria.

As in [28] and [29], we focus on symmetric 16-state encoders. The feedback tap coefficients are $\left\{f_{0}, f_{1}, f_{2}, f_{3}, f_{4}\right\}$. We always have $f_{0}=1$, since it provides the tap for the encoder input. Denote the feedback polynomial as $F(D)=f_{0}+f_{1} D+f_{2} D^{2}+$ $f_{3} D^{3}+f_{4} D^{4}$, and the two feed-forward polynomials as $G(D)$ and $H(D)$, respectively. Then for such an RNSC encoder, there are altogether $2^{4} \times\left(2^{5}-1\right) \times\left(2^{5}-1\right)=15376$ possible combinations; an exhaustive search over the entire code space is clearly not feasible. However, the two asymptotic properties studied in Section III serve as good design criteria to eliminate poor encoder structures.

First, as proved by Theorem 2 in Section III, we choose to have the last feedback tap coefficient $f_{4}=1$ to fully exploit the encoder memory. For the feed-forward polynomials, having $G(D)=1$ or $G(D)=D^{m}$, where $m=1, \ldots, 4$, yields the same performance, since the only difference is a shift of time delay. The same holds for having $G(D)=1+D^{2}$ or $G(D)=D^{2}+D^{4}$. Therefore, without loss of generality, we can choose to fix the first tap coefficient of both feed-forward polynomials to one. Furthermore, for obvious reasons, we do not want the two feed-forward polynomials to be identical. Then the total number of possible encoders in our search space is reduced down to $2^{3} \times 2^{4} \times\left(2^{4}-1\right)=1920$, which is still impractical for an exhaustive search. Second, according to Theorem 1, we should only consider the choices of $G(D)$ and $F(D)$ such that $G(D)$ is not divisible by $F(D)$. Furthermore, by our empirically verified conjecture, given $f_{4}=1$, the selection of relatively prime $F(D)$ and $G(D)$ can guarantee that the encoder output has an asymptotically uniform fourth-order distribution. Thus, additional inferior candidate encoder structures can be eliminated. Finally, again to avoid an exhaustive search, we take advantage of the optimization results found in STCs [29]; i.e., we first find the best pair of $F(D)$ and $G(D)$, and then search for the best second feed-forward polynomial $H(D)$. The iterative search procedure for (sub)optimal encoder structures, given a source distribution, is implemented as follows.

1) Using an STC, fix the feed-forward polynomial $G(D)=1$ and search for the best feedback polynomial $F(D)$ with $f_{0}=$ $f_{4}=1$.

2) With the $F(D)$ found in step 1), search for the best $G(D)$ among all remaining possible choices, with the condition that the greatest common divisor of $F(D)$ and $G(D)$ is 1 .

3) With the $G(D)$ found in step 2), go back to step 1). If $F(D)$ coincides with the one obtained in step 1), go to step 4); otherwise, proceed to step 2).

4) For an NSTC, fix the pair ( $F(D), G(D))$ found above and search for the best second feed-forward polynomial $H(D)$.

\section{B. Decoder Design}

When RNSC encoders are used as constituent encoders, unlike in STCs, the a posteriori $\log$-likelihood ratio $\Lambda\left(U_{k}\right)$ in the turbo decoder [23], [43] can only be decomposed into two terms

$$
\Lambda\left(U_{k}\right)=L_{\mathrm{ex}}\left(U_{k}\right)+L_{\mathrm{ap}}\left(U_{k}\right)
$$


where the new extrinsic term involves two parity sequences. For AWGN channels, we have

$$
L_{\mathrm{ex}}\left(U_{k}\right)=\log \frac{\sum_{s} \sum_{s^{\prime}} \gamma\left(y_{k}^{h}, y_{k}^{g} \mid 1, s, s^{\prime}\right) \cdot \alpha_{k-1}\left(s^{\prime}\right) \cdot \beta_{k}(s)}{\sum_{s} \sum_{s^{\prime}} \gamma\left(y_{k}^{h}, y_{k}^{g} \mid 0, s, s^{\prime}\right) \cdot \alpha_{k-1}\left(s^{\prime}\right) \cdot \beta_{k}(s)}
$$

where the summations for $s$ and $s^{\prime}$ are both over all $2^{M}$ possible states, and for $i=0,1$

$$
\begin{aligned}
\gamma\left(y_{k}^{h}, y_{k}^{g} \mid i, s, s^{\prime}\right)=p\left(y_{k}^{h} \mid U_{k}=i, S_{k}=s\right) \\
\cdot p\left(y_{k}^{g} \mid U_{k}=i, S_{k}=s\right) \cdot \operatorname{Pr}\left\{U_{k}=i \mid S_{k}=s, S_{k-1}=s^{\prime}\right\}
\end{aligned}
$$

where $S_{k}$ is the encoder state at time $k$, and $y_{k}^{h}$ and $y_{k}^{g}$ are the noise-corrupted version of $x_{k}^{h}$ and $x_{k}^{g}$, which are the parity bits generated from the two feed-forward polynomials.

For Rayleigh fading channels, the extrinsic term needs to be modified to appropriately incorporate the channel statistics. The extrinsic term therefore becomes

$L_{\mathrm{ex}}\left(U_{k}\right)=\log \frac{\sum_{s, s^{\prime}} \gamma\left(y_{k}^{h}, y_{k}^{g} \mid 1, s, s^{\prime}, a_{k}^{h}, a_{k}^{g}\right) \cdot \alpha_{k-1}\left(s^{\prime}\right) \cdot \beta_{k}(s)}{\sum_{s, s^{\prime}} \gamma\left(y_{k}^{h}, y_{k}^{g} \mid 0, s, s^{\prime}, a_{k}^{h}, a_{k}^{g}\right) \cdot \alpha_{k-1}\left(s^{\prime}\right) \cdot \beta_{k}(s)}$

where for $i=0,1$

$$
\begin{aligned}
& \gamma\left(y_{k}^{h}, y_{k}^{g} \mid i, s, s^{\prime}, a_{k}^{h}, a_{k}^{g}\right)=p\left(y_{k}^{h} \mid U_{k}=i, S_{k}=s, a_{k}^{h}\right) \\
& \quad \cdot p\left(y_{k}^{g} \mid U_{k}=i, S_{k}=s, a_{k}^{g}\right) \cdot \operatorname{Pr}\left\{U_{k}=i \mid S_{k}=s, S_{k-1}=s^{\prime}\right\}
\end{aligned}
$$

where $a_{k}^{h}$ and $a_{k}^{g}$ are the fading factors. When the source is nonuniform i.i.d., $\log \left(\left(1-p_{0}\right) / p_{0}\right)$ is used as the initial a priori input $L_{\mathrm{ap}}\left(U_{k}\right)$ to the first decoder at the first iteration; then at the following iterations, $L_{\mathrm{ex}}+\log \left(\left(1-p_{0}\right) / p_{0}\right)$ is used as the new extrinsic information for both constituent decoders at each iteration.

\section{NUMERICAL RESULTS AND DISCUSSION}

In this section, we present simulation results of our NSTCs for uniform i.i.d. sources over BPSK-modulated AWGN and Rayleigh fading channels with known CSI. ${ }^{3}$ In the following, all NSTCs adopt the decoder discussed in Section IV-B, while all systematic codes employ the BCJR algorithm with the modification proposed in [29].

\section{A. Performance Evaluations}

The performance is measured in terms of the BER versus $E_{b} / N_{0}$, where $E_{b}=E_{s} / R_{c}$ is the average energy per source bit. All simulated turbo codes have 16-state constituent encoders

\footnotetext{
${ }^{3}$ It is assumed throughout that the decoder has perfect knowledge of the source distribution $p_{0}$. Simulations on the effect of mismatch in $p_{0}$ show little performance loss if the value of $p_{0}$ used at the decoder is within a reasonable distance from the true $p_{0}$. For example, when the true distribution is $p_{0}=$ 0.9 and the decoder assumes it is 0.8 , the performance degradation is no more than $0.1 \mathrm{~dB}$. Furthermore, information on $p_{0}$ can be sent to the receiver as an overhead with negligible bandwidth loss. Finally, if no overhead information is sent, $p_{0}$ can possibly be estimated at the decoder (e.g., see [27] where the source statistics are estimated at the receiver in the context of turbo decoding of hidden Markov sources).
}

and use the same pseudorandom interleaver introduced in [23]. The sequence length is $N=512 \times 512=262144$ and at least 200 blocks are simulated; this would guarantee a reliable BER estimation at the $10^{-5}$ level with 524 errors. The number of iterations employed in the decoder is 20; note that additional iterations result in minor improvements. All results are presented for turbo codes with structure b) encoders (see Fig. 3), as they provide a better performance than codes with structure a) encoders. Simulations are performed for rates $R_{c}=1 / 3$ and $R_{c}=1 / 2$ with $p_{0}=0.8$ and 0.9 . From our simulations, for both rates $1 / 3$ and $1 / 2$, the best found RNSC encoder structure for $p_{0}=0.8$ and for both channels has each constituent encoder with feedback polynomial 35 and feed-forward polynomials 23 and 25; this is denoted by the octal triplet $(35,23,25)$. For $p_{0}=0.9$, the best structure is $(31,23,27)$. Several other encoders give very competitive performance, such as $(35,23,31)$ for $p_{0}=0.8,(31,23,35)$ and $(31,23,37)$ for $p_{0}=0.9$. We hereafter characterize all NSTCs by triplets as described above (while their systematic peers are represented by the conventional octal pair).

Fig. 4 shows the performance over AWGN channels of our rate-1/3 NSTCs in comparison with their systematic peers investigated in [28] and [29], as well as with Berrou's $(37,21)$ code, which offers the best waterfall performance (among 16-state encoders) for uniform i.i.d. sources. At the $10^{-5}$ BER level, when $p_{0}=0.8$, our $(35,23,25)$ NSTC offers a $0.45-\mathrm{dB}$ gain over its $(35,23)$ systematic peer; when $p_{0}=0.9$, our $(31,23,27)$ code offers an improvement of $0.89 \mathrm{~dB}$ over the systematic $(31,23)$ code. ${ }^{4}$ In comparison with Berrou's $(37,21)$ code performance, the gains achieved by exploiting the source redundancy and encoder optimization are, therefore, $1.48 \mathrm{~dB}$ and $3.25 \mathrm{~dB}$ for $p_{0}=$ 0.8 and 0.9 , respectively.

Fig. 5 shows similar results over AWGN channels for rate$1 / 2$. In this case, the gains are generally more significant. In comparison with the best STCs, at the $10^{-5}$ BER level, for $p_{0}=0.8$ and 0.9 , the gains achieved are 0.69 and $1.56 \mathrm{~dB}$, respectively. Furthermore, the gains over Berrou's code due to combining the optimized encoder with the modified decoder that exploits the source redundancy are $1.57 \mathrm{~dB}\left(p_{0}=0.8\right)$ and $3.72 \mathrm{~dB}\left(p_{0}=0.9\right)$.

Simulations over Rayleigh channels are also provided in Figs. 6 and 7. For rate- $1 / 3$ codes, at a $10^{-5}$ BER, when $p_{0}=0.8$, our $(35,23,25)$ NSTC provides a $0.40 \mathrm{~dB}$ gain over its $(35,23)$ systematic peer; when $p_{0}=0.9$, the improvement is $1.01 \mathrm{~dB}$ with encoder structure $(31,23,27)$. In comparison with Berrou's $(37,21)$ code, the gains achieved by exploiting the source redundancy and encoder optimization are 1.76 and $3.87 \mathrm{~dB}$ for $p_{0}=0.8$ and 0.9 , respectively. For rate- $1 / 2$, as shown in Fig. 7, the gains are more pronounced. In comparison

\footnotetext{
${ }^{4}$ It should also be indicated that our nonsystematic joint source-channel turbo codes maintain a similar level of performance gains over their systematic peers when the sequence length is shorter than 262144 . For example, for a sequence length of $N=128 \times 128=16384$, with $p_{0}=0.9$ and the same rate and channel conditions as in Fig. 4, our $(31,23,27)$ nonsystematic code offers around $0.9 \mathrm{~dB}$ gain over its $(31,23)$ systematic peer; a similar gain is obtained for $N=32 \times 32=1024$. These gains are interesting to note for practical situations where delay may be limited.
} 


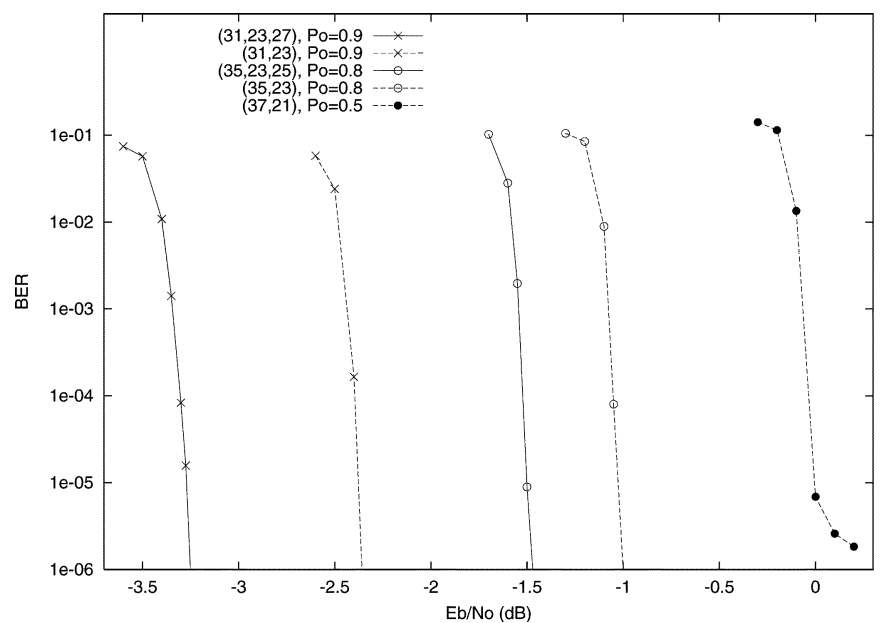

Fig. 4. Turbo codes for nonuniform i.i.d. sources, $R_{c}=1 / 3, N=262144$, AWGN channel.

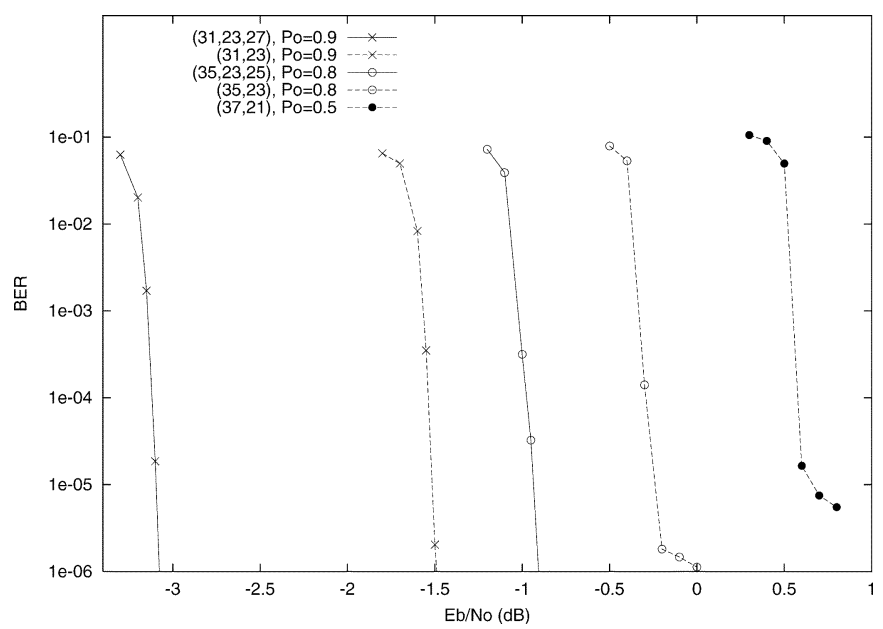

Fig. 5. Turbo codes for nonuniform i.i.d. sources, $R_{c}=1 / 2, N=262144$, AWGN channel.

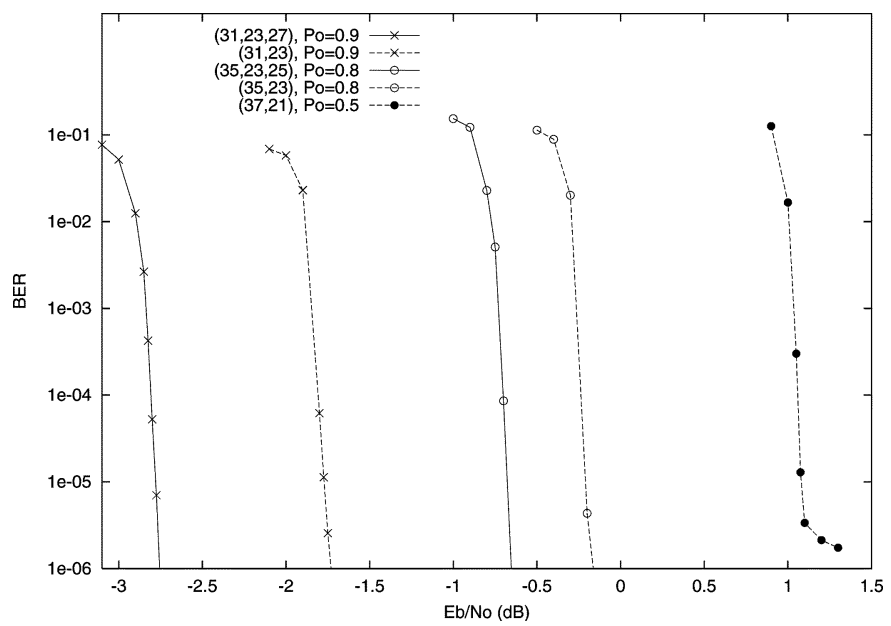

Fig. 6. Turbo codes for nonuniform memoryless sources, $R_{c}=1 / 3, N=$ 262144 , Rayleigh fading channel.

with the best STCs, at a $10^{-5} \mathrm{BER}$, for $p_{0}=0.8$ and 0.9 , the gains are 0.77 and $1.84 \mathrm{~dB}$, respectively. Furthermore, the gains due to combining the optimized encoder with the modified decoder are $2.01 \mathrm{~dB}$ (for $p_{0}=0.8$ ) and $4.71 \mathrm{~dB}$ (for $p_{0}=0.9$ ).

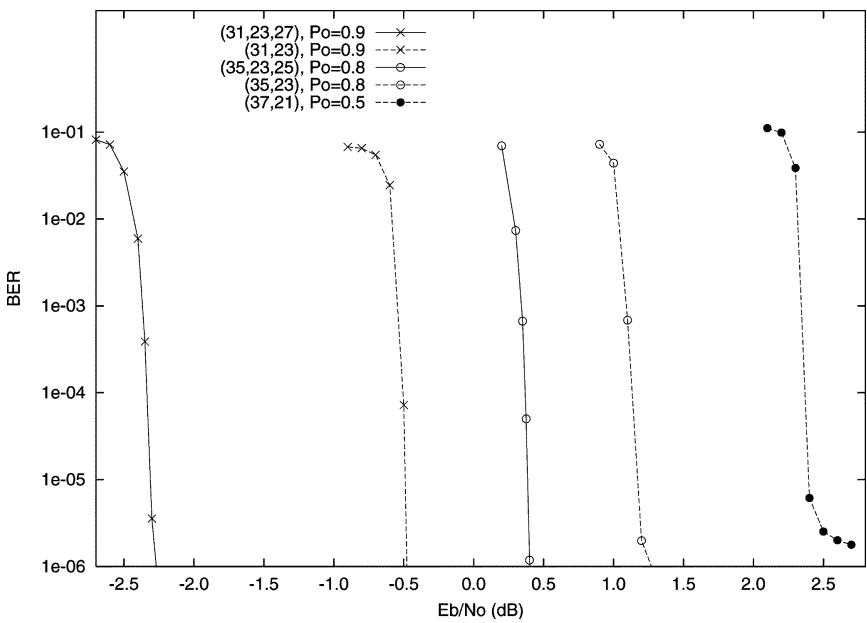

Fig. 7. Turbo codes for nonuniform memoryless sources, $R_{c}=1 / 2, N=$ 262144 , Rayleigh fading channel.

To achieve a desired rate via puncturing, using different puncturing patterns may result in different performances. For example, when structure b) is used for an overall rate of $1 / 3$, we may choose to puncture $1 / 4$ of each parity sequence according to various patterns, or we may puncture half of two parity sequences, and leave the other two sequences intact. Simulations show the best puncturing pattern is to keep the parity sequence generated by feed-forward polynomial 23 intact and puncture half of the one generated by the other feed-forward polynomial. The performance of this puncturing pattern is about $0.2 \mathrm{~dB}$ better than other patterns; in particular, it is $0.3 \mathrm{~dB}$ better than the performance offered by structure a). For an overall rate of $1 / 2$, structure b) is also better than a), and the best puncturing pattern is to delete all even (odd) position bits of the sequences generated by feed-forward polynomial 23 , and delete all odd (even) position bits of the sequences generated by the other feed-forward polynomial.

In previous work on NSTCs for uniform i.i.d. sources, it is verified via extensive simulations that most nonsystematic codes show inferior performance to their systematic peers [36]-[38], except for the one in [38] employing a nonsystematic "quick-look-in" constituent code, which is basically "close" to a systematic code. Also, it is pointed out in [37] and [38] that at low values of $E_{b} / N_{0}$, the initial extrinsic estimates for the information bits provided by NSTCs are not as good as those of STCs, due to the lack of received channel values; this motivates their choice of a close-to-systematic code in the nonsystematic family. However, as shown by our results, STCs are not a suitable choice for heavily biased nonuniform sources; therefore, close-to-systematic codes may not be desired in this case. Another encoder structure called "big-numerator-little-denominator" [35], which provides gains over Berrou's code for uniform sources, may also be unsuitable for nonuniform sources, since the denominator results in nonuniform higher order output distributions.

In the scenario of nonuniform i.i.d. sources, there are two factors playing against each other, the a priori knowledge of $p_{0}$ at the decoder, and the systematic structure. When the source is heavily biased, systematic codes considerably underperform 
TABLE II

OPTA GAPS FOR STCS AND NSTCS IN $E_{b} / N_{0}(\mathrm{DB})$ AT BER $=10^{-5}$

\begin{tabular}{|c|c|c|c|c|c|}
\hline Channel & $R_{c}$ & $p_{0}$ & OPTA & STC OPTA gaps [29] & NSTC OPTA gaps \\
\hline \multirow{4}{*}{$\begin{array}{l}\text { AWGN } \\
\text { channel }\end{array}$} & \multirow[t]{2}{*}{$1 / 3$} & 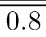 & -2.24 & $\bar{~} 1.19$ & 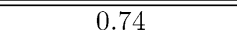 \\
\hline & & 0.9 & -4.40 & 2.02 & 1.13 \\
\hline & \multirow[t]{2}{*}{$1 / 2$} & 0.8 & -1.81 & 1.56 & 0.87 \\
\hline & & 0.9 & -4.14 & 2.61 & 1.05 \\
\hline \multirow{4}{*}{$\begin{array}{c}\text { Rayleigh } \\
\text { channel }\end{array}$} & \multirow[t]{2}{*}{$1 / 3$} & 0.8 & -1.56 & 1.28 & 0.88 \\
\hline & & 0.9 & -3.96 & 2.18 & 1.17 \\
\hline & \multirow[t]{2}{*}{$1 / 2$} & 0.8 & -0.73 & 1.88 & 1.11 \\
\hline & & 0.9 & -3.47 & 2.99 & 1.15 \\
\hline
\end{tabular}

due to the distribution mismatch between the source and the capacity-achieving channel input. Furthermore, the knowledge of a biased $p_{0}$ can give good initial extrinsic estimations of the information bits at the decoder, thus eliminating the need for the systematic structure. This may explain why NSTCs, which do not suffer from any distribution mismatch (at least asymptotically), offer superior performance over their systematic counterparts. On the other hand, when the source distribution is close to uniform, the distribution mismatch due to a systematic structure becomes minor. Furthermore, very little useful knowledge about the information bits is provided from $p_{0}$ at the decoder, while a systematic structure, even when its bits are received corrupted at the receiver due to channel noise, provides more reliable extrinsic estimations in the initial iterative decoding stages for low $E_{b} / N_{0}$. In particular, as pointed out in [36] and [37], when the source is exactly uniform, there is no useful knowledge from $p_{0}$ at all, and thus, the systematic structure plays a critically important role. To investigate the role of the systematic bits for less biased nonuniform i.i.d. sources, we also study the performance of so-called "extended" STCs. The results are briefly summarized in the following.

The encoder of an "extended" STC consists of one systematic output and two RNSC encoders, each of which produces two parity outputs. The overall rate is, therefore, $1 / 5$. Higher rates (e.g., 1/3 and 1/2) are obtained by partial puncturing of the systematic part and partial puncturing of the four parity sequences. For $p_{0}=0.6,0.7$, and 0.8 , and for $R_{c}=1 / 2$ and $1 / 3$, we performed simulations using the following puncturing patterns. Delete $j / 8$ of the systematic sequence, where $j=0,1, \ldots, 8$, and delete evenly the four parity sequences to maintain the desired overall rates. Our simulations demonstrate that when $p_{0}=$ 0.7 , starting from $j=0$, the performance is improved as $j$ increases up to $j=4$, which yields approximately a $0.1 \mathrm{~dB}$ gain in $E_{b} / N_{0}$ at the $10^{-5}$ BER level over the system with pattern $j=0$. Then the performance degrades as $j$ increases from four to eight. Therefore, when $p_{0}=0.7$, puncturing half of the systematic sequence yields the best performance. When $p_{0}=0.8$, we observe a monotonic performance improvement as $j$ increases from zero up to eight, which indicates that purely NSTCs should be preferred. When $p_{0}=0.6$, we observe exactly the opposite behavior: preserving all systematic bits gives the best performance. These observations indicate, as remarked in [35]-[38], that a systematic encoding structure is especially important when not enough a priori knowledge about the source is available at the decoder.

\section{B. Shannon Limit}

Shannon's Lossy Joint Source-Channel Coding Theorem states that, for a given memoryless source and channel pair ${ }^{5}$ and for sufficiently large source-block lengths, the source can be transmitted via a source-channel code over the channel at a transmission rate of $R_{c}$ source symbols/channel symbol and reproduced at the receiver end within an end-to-end distortion given by $\mathrm{D}$ if the following condition is satisfied [32]:

$$
R_{c} \cdot R(\mathrm{D})<C
$$

where $C$ is the channel capacity and $R(\mathrm{D})$ is the source rate-distortion function. For a discrete binary nonuniform i.i.d. source with distribution $p_{0}$, we have that $\mathrm{D}=P_{e}$ (the BER) under the Hamming distortion measure; then $R(\mathrm{D})$ becomes

$$
R\left(P_{e}\right)= \begin{cases}h_{b}\left(p_{0}\right)-h_{b}\left(P_{e}\right), & 0 \leq P_{e} \leq \min \left\{p_{0}, p_{1}\right\} \\ 0, & P_{e}>\min \left\{p_{0}, p_{1}\right\}\end{cases}
$$

where $p_{1}=1-p_{0}$, and $h_{b}(x)=-x \log _{2} x-(1-x) \log _{2}(1-x)$ is the binary entropy function.

As seen in Section II, the capacity of an AWGN or Rayleigh channel is a function of SNR $E_{s} / N_{0}$, or equivalently, $E_{b} / N_{0}$ (where $E_{b}=E_{s} / R_{c}$ ); therefore, the optimum value of $E_{b} / N_{0}$ to guarantee a BER of $P_{e}$, called the Shannon limit or OPTA, can be solved using (3), assuming equality. The Shannon limit cannot be explicitly solved for our BPSK-modulated channels due to the lack of a closed-form expression, so it is computed via numerical integration.

For the simulations of the above subsection, the OPTA values at the $10^{-5}$ BER level are computed. The OPTA gaps, which are the distances between our system performance and the corresponding OPTA values, are provided in Table II. We observe that the OPTA gaps are significantly reduced by NSTCs. For example, for AWGN channels and $R_{c}=1 / 2$, when $p_{0}=$ 0.8 , STCs provide a performance which is $1.56 \mathrm{~dB}$ away from OPTA; on the other hand, for our NSTCs, the OPTA gap is $0.87 \mathrm{~dB}$. When $p_{0}=0.9$, the OPTA gap is reduced from 2.61 to $1.05 \mathrm{~dB}$.

\section{COMPARISON WITH TANDEM SCHEMES}

Traditionally, source and channel coding are designed separately, resulting in a so-called tandem coding scheme. That is,

\footnotetext{
${ }^{5}$ The above theorem also holds for wider classes of sources and channels with memory (e.g., stationary ergodic sources and channels with additive stationary ergodic noise) [31].
} 
the source is compressed first, and then channel coded. This is justified by Shannon's separation principle [44], which states that there is no loss of optimality in such separation as long as unlimited coding delay and complexity are available. However, in practice, joint source-channel coding often outperforms traditional tandem coding when delay and system complexity are constrained.

We next compare the performance of our joint source-channel system with that of two tandem schemes for the same overall transmission rate. Each tandem scheme consists of a fourth-order Huffman code followed by a rate $R_{c}=1 / 3$ turbo code. The overall rate for both tandem and joint source-channel coding systems is $r=R_{c} / R_{s}=1 / 2$ source symbol/channel symbol (in the joint coding scheme, $R_{c}=1 / 2$ and $R_{s}=1$ since no source coding is performed). Therefore, the Huffman code needs to be of rate $R_{s}=2 / 3$ code bits/source symbol. Since the average rate of the Huffman code depends on the source distribution, we need to find the value of $p_{0}$ which renders the Huffman code rate (not the entropy) $R_{s}=2 / 3$ code bits/source symbol with satisfactory accuracy. By using the bisection method, we obtain that when $p_{0}=0.83079$, a fourth-order Huffman code has rate $R_{s}=0.666668$ code bits/source symbol. Therefore, simulations are performed for this value of $p_{0}$.

Berrou's pseudorandom interleaver [23] requires the sequence length to be an even power of two; this inflexibility is an obstacle in the design of the tandem scheme, since the Huffman code is a variable-length code. The $S$-random interleaver [45], however, can take an input sequence of arbitrary length, and it yields good BER performance. We thus adopt the $S$-random interleaver in the turbo code. We also do not terminate the first constituent turbo encoder, because otherwise, errors in the tail bits of the turbo-decoded sequence would introduce irrecoverable errors in the Huffman-decoded sequence. For fair comparison, our system also uses the $S$-random interleaver, and its first constituent encoder is not terminated.

The tandem scheme is implemented as follows: 1) the source generates a nonuniform i.i.d. sequence with length $N$, and $p_{0}=0.83079 ; 2$ ) the Huffman encoder produces a compressed sequence with variable length, whose mean is approximately $(2 / 3) N$; 3$)$ an $S$-random interleaver is generated for this given length; 4) the sequence is turbo encoded using the $S$-random interleaver generated in 3); 5) the sequence is BPSK modulated and transmitted over a Rayleigh fading channel; 6) the sequence is turbo decoded and then Huffman decoded.

Another issue is the choice of the source sequence length $N$. Due to error propagation in the Huffman decoder, a few errors in the turbo-decoded sequence could result in a big percentage of errors in the final Huffman-decoded sequence. Furthermore, what matters is not only the number of errors in the turbo-decoded sequence, but also the positions of the erroneous bits. This is due to the fact that an error occurring in the first few bits of the turbo-decoded sequence has a much longer propagating effect than an error occurring in the tail bits. Therefore, a sufficiently large number of blocks is necessary to obtain a good average performance. After several tests, we chose to use $N=12000$ and 60000 blocks.

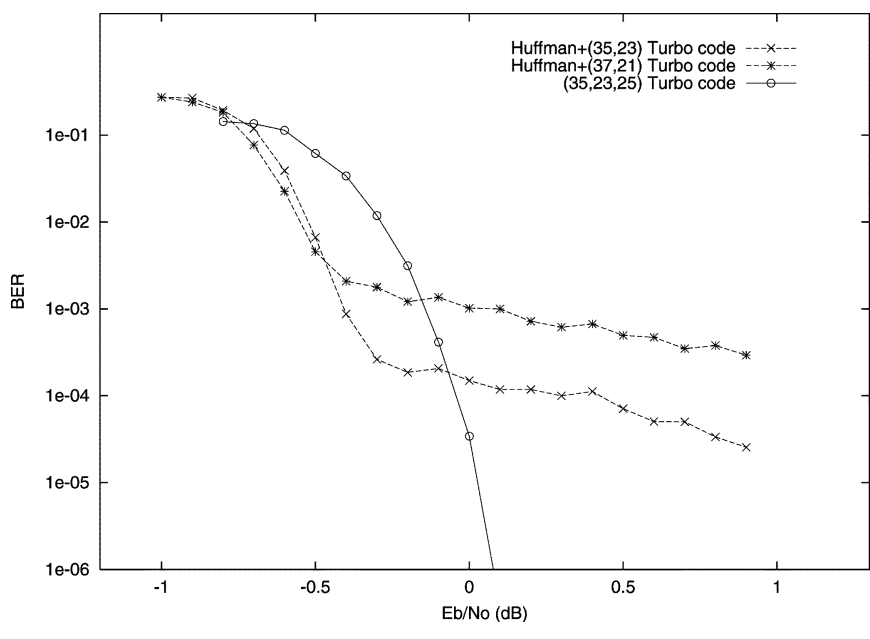

Fig. 8. Performance comparison of our system $\left(R_{s}=1, R_{c}=1 / 2\right)$ with that of tandem schemes $\left(R_{s}=2 / 3, R_{c}=1 / 3\right), p_{0}=0.83079, N=12000$, Rayleigh fading channel.

For a given $N$, the larger the "spread" $S$ of the $S$-random interleaver is, the better is the performance. However, in practice, generating an $S$-random interleaver with a large $S$ requires a substantial amount of computing time, and sometimes such an interleaver may not be successfully generated. Thus, in order to reduce the computation time and to guarantee the successful generation of $S$-random interleavers of arbitrary size, $S$ is set to equal 10 .

Fig. 8 shows the performance of our system versus that of two tandem schemes over Rayleigh channels. Twenty iterations are used in the turbo decoder. In the first tandem scheme, the turbo code with $R_{c}=1 / 3$ is Berrou's $(37,21)$ code, which offers an excellent waterfall performance for uniform sources among all 16-state codes. However, due to a relatively high error floor provided by Berrou's code, this tandem scheme suffers from a high-BER performance caused by error propagation in the Huffman decoder. Thus, we also evaluate a second tandem scheme using the $(35,23)$ turbo code, which has a significantly lower error floor at the expense of a slight waterfall performance loss. Although at very high BERs both tandem schemes are better than our system, their error floors occur at high BERs $\left(10^{-3}\right.$ for the $(37,21)$ code, and $10^{-4}$ for the $(35,23)$ code). Therefore, at low BER levels, our system offers superior performance over both tandem schemes. Interestingly, most traditional joint source-channel coding schemes outperform tandem schemes at high BER levels (e.g., [2], [3], and [7]), while in the context of turbo codes, the opposite result is observed. Alternative source-channel coding systems using jointly designed Huffman and turbo codes have been recently proposed in [46] and [47]. It would be interesting to make performance comparisons with these schemes. However, our system has lower complexity, since the source encoding and decoding parts are omitted.

\section{CONCLUSION}

In this paper, the joint source-channel coding issue of transmitting nonuniform memoryless sources via turbo codes over AWGN and Rayleigh channels is investigated. Necessary and 
sufficient conditions are proved for recursive convolutional encoders having an asymptotically uniform state and marginal output distributions, regardless of the degree of source nonuniformity. Therefore, recursive nonsystematic turbo source-channel codes are proposed, and the outputs of our selected codes are suitably matched to the channel input as they nearly maximize the channel mutual information. Simulation results demonstrate substantial coding gains (up to $1.84 \mathrm{~dB}$ ) over STCs designed in [28] and [29], and the OPTA gaps are significantly reduced. Finally, our system is compared with two tandem schemes, which employ a near-optimal Huffman code followed by a standard turbo code. Our system offers substantially better performance at low BERs, and enjoys a lower complexity.

\section{ACKNOWLEDGMENT}

The authors would like to thank the anonymous reviewers for their valuable suggestions, which were very helpful in improving the presentation of this paper.

\section{REFERENCES}

[1] P. Burlina, F. Alajaji, and R. Chellappa, "Transmission of two-tone images over noisy communication channels with memory," Univ. of Maryland, Center for Automat. Res., College Park, MD, Tech. Rep. CAR-TR814, 1996.

[2] J. Kroll and N. Phamdo, "Source-channel optimized trellis codes for bitonal image transmission over AWGN channels," IEEE Trans. Image Processing, vol. 8, pp. 899-912, July 1999.

[3] F. Alajaji, N. Phamdo, and T. Fuja, "Channel codes that exploit the residual redundancy in CELP-encoded speech," IEEE Trans. Speech Audio Processing, vol. 4, pp. 325-336, Sept. 1996.

[4] R. B. Blizard, "Convolutional coding for data compression," Martin Marietta Corp., Denver, CO, Rep. R-69-17, 1969.

[5] V. N. Koshelev, "Direct sequential encoding and decoding for discrete sources," IEEE Trans. Inform. Theory, vol. IT-19, pp. 340-343, May 1973.

[6] M. E. Hellman, "Convolutional source encoding," IEEE Trans. Inform. Theory, vol. IT-21, pp. 651-656, Nov. 1975.

[7] F. Alajaji, N. Phamdo, N. Farvardin, and T. Fuja, "Detection of binary Markov sources over channels with additive Markov noise," IEEE Trans. Inform. Theory, vol. 42, pp. 230-239, Jan. 1996.

[8] T. Fingscheidt, T. Hindelang, R. V. Cox, and N. Seshadri, "Joint source-channel (de)coding for mobile communications," IEEE Trans. Commun., vol. 50, pp. 200-212, Feb. 2002.

[9] J. Hagenauer, "Source-controlled channel decoding," IEEE Trans. Commun., vol. 43, pp. 2449-2457, Sept. 1995.

[10] F. Lahouti and A. Khandani, "Sequence MMSE source decoding over noisy channels using the residual redundancies," in Proc. Allerton Conf. Communications, Control, Computing, Monticello, IL, Oct. 2001, pp. 1306-1315.

[11] M. Park and D. Miller, "Improved image decoding over noisy channels using minimum mean-squared estimation and a Markov mesh," IEEE Trans. Image Processing, vol. 8, pp. 863-867, June 1999.

[12] _ - "Joint source-channel decoding for variable-length encoded data by exact and approximate MAP sequence estimation," IEEE Trans. Commun., vol. 48, pp. 1-6, Jan. 2000.

[13] M. Adrat, P. Vary, and J. Spittka, "Iterative source-channel decoding using extrinsic information of softbit-source decoding," in Proc. IEEE Int. Conf. Acoustics, Speech, Signal Processing, Salt Lake City, UT, May 2001, pp. 2653-2656.

[14] N. Görtz, "On the iterative approximation of optimal joint sourcechannel decoding," IEEE J. Select. Areas Commun., vol. 19, pp. 1662-1670, Sept. 2001
[15] N. Phamdo, "Quantization over discrete noisy channels under complexity constraints," Ph.D. dissertation, Univ. Maryland, College Park, MD, 1993.

[16] N. Phamdo and N. Farvardin, "Optimal detection of discrete Markov sources over discrete memoryless channels-Applications to combined source-channel coding," IEEE Trans. Inform. Theory, vol. 40, pp. 186-193, Jan. 1994

[17] - "Scalar quantization of memoryless sources over memoryless channels using rate-one convolutional codes," in Proc. Int. Symp. Information Theory, Trondheim, Norway, June 1994, p. 235.

[18] K. Sayood and J. C. Borkenhagen, "Use of residual redundancy in the design of joint source-channel codes," IEEE Trans. Commun., vol. 39, pp. 838-846, June 1991 .

[19] M. Skoglund, "Soft decoding for vector quantization over noisy channels with memory," IEEE Trans. Inform. Theory, vol. 45, pp. 1293-1307, May 1999

[20] - "Bit-estimate-based decoding for vector quantization over noisy channels with intersymbol interference," IEEE Trans. Commun., vol. 48 pp. 1309-1317, Aug. 2000.

[21] R. E. Van Dyck and D. Miller, "Transport of wireless video using separate, concatenated, and joint source-channel coding," Proc. IEEE, vol 87, pp. 1734-1750, Oct. 1999.

[22] W. Xu, J. Hagenauer, and J. Hollmann, "Joint source-channel decoding using the residual redundancy in compressed images," in Proc. Int. Conf. Communications, Dallas, TX, June 1996, pp. 142-148.

[23] C. Berrou and A. Glavieux, "Near-optimum error-correcting coding and decoding: Turbo codes," IEEE Trans. Commun., vol. 44, pp. 1261-1271, Oct. 1996.

[24] E. K. Hall and S. G. Wilson, "Design and analysis of turbo codes on Rayleigh fading channels," IEEE J. Select. Areas Commun., vol. 16, pp 160-174, Feb. 1998

[25] R. J. McEliece, "Are turbo-like codes effective on nonstandard channels?," IEEE Inform. Theory Soc. Newsletter, vol. 51, pp. 1-8, Dec. 2001.

[26] J. Garcia-Frias and J. D. Villasenor, "Combining hidden Markov source models and parallel concatenated codes," IEEE Commun. Lett., vol. 1 pp. 111-113, July 1997.

[27] _ "Joint turbo decoding and estimation of hidden Markov sources," IEEE J. Select. Areas Commun., vol. 19, pp. 1671-1679, Sept. 2001.

[28] G.-C. Zhu and F. Alajaji, "Design of turbo codes for nonequiprobable memoryless sources," in Proc. 39th Allerton Conf. Communication Control, Computing, Monticello, IL, Oct. 2001, pp. 1253-1262.

[29] — - "Turbo codes for nonuniform memoryless sources over noisy channels," IEEE Commun. Lett., vol. 6, pp. 64-66, Feb. 2002.

[30] S. Shamai and S. Verdú, "The empirical distribution of good codes," IEEE Trans. Inform. Theory, vol. 43, pp. 836-846, May 1997.

[31] T. Berger, Rate Distortion Theory: A Mathematical Basis for Data Compression. Englewood Cliffs, NJ: Prentice-Hall, 1971.

[32] R. J. McEliece, The Theory of Information and Coding, 2nd ed. Reading, MA: Addison-Wesley, 2002.

[33] I. J. Fair, V. K. Bhargava, and Q. Wang, "On the power spectral density of self-synchronizing scrambled sequences," IEEE Trans. Inform. Theory, vol. 44, pp. 1687-1693, July 1998.

[34] D. G. Leeper, "A universal digital data scrambler," Bell Syst. Tech. J., vol. 52, no. 10, pp. 1851-1865, Dec. 1973.

[35] D. J. Costello, Jr., P. C. Massey, O. M. Collins, and O. Y. Takeshita, "Some reflections on the mythology of turbo codes," in Proc. 3rd ITG Conf. Source-Channel Coding, Munich, Germany, Jan. 2000, pp. $157-160$.

[36] O. M. Collins, O. Y. Takeshita, and D. J. Costello, Jr., "Iterative decoding of nonsystematic turbo codes," in Proc. Int. Symp. Information Theory, Sorrento, Italy, June 2000, p. 172.

[37] D. J. Costello, Jr., H. A. Cabral, and O. Y. Takeshita, "Some thoughts on the equivalence of systematic and nonsystematic convolutional encoders," in Codes, Graphs and Systems, R. E. Blahut and R. Koetter, Eds. Norwell, MA: Kluwer, 2002.

[38] P. C. Massey and D. J. Costello, Jr., "Turbo codes with recursive nonsystematic quick-look-in constituent codes," in Proc. Int. Symp. Information Theory, Washington, DC, June 2001, p. 141.

[39] L. R. Bahl, J. Cocke, F. Jelinek, and J. Raviv, "Optimal decoding of linear codes for minimizing symbol error rate," IEEE Trans. Inform. Theory, vol. IT-20, pp. 248-287, Mar. 1974 
[40] S. Benedetto and G. Montorsi, "Unveiling turbo codes: Some results on parallel concatenated coding schemes," IEEE Trans. Inform. Theory, vol. 42, pp. 409-428, Mar. 1996.

[41] T. M. Duman and M. Salehi, "New performance bounds for turbo codes," IEEE Trans. Commun., vol. 46, pp. 717-723, June 1998.

[42] S. Shamai and I. Sason, "Variations on Gallager's bounding techniques: Performance bounds for turbo codes in Gaussian and fading channels," in Proc. 2nd Int. Symp. Turbo Codes and Related Topics, Brest, France, Sept. 2000, pp. 27-34.

[43] J. Hagenauer, E. Offer, and L. Papke, "Iterative decoding of binary block and convolutional codes," IEEE Trans. Inform. Theory, vol. 42, pp. 429-445, Mar. 1996.

[44] C. E. Shannon, "A mathematical theory of communication," Bell Syst. Tech. J., vol. 27, pp. 379-423 and 623-656, 1948.

[45] D. Divsalar and F. Pollara, "Multiple turbo codes for deep-space communications," Jet Propulsion Lab., Pasadena, CA, TDA Prog. Rep. 42-121, 1995.

[46] L. Guivarch, J.-C. Carlach, and P. Siohan, "Joint source-channel soft decoding of Huffman codes with turbo codes," in Proc. Data Compression Conf., Snowbird, UT, Mar. 2000, pp. 83-92.

[47] J. Hagenauer and R. Bauer, "The turbo principle in joint source-channel decoding of variable-length codes," in Proc. IEEE Information Theory Workshop, Cairns, Australia, Sept. 2001, pp. 128-130.

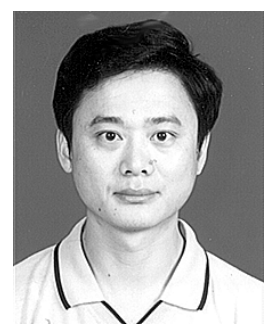

Guang-Chong Zhu (M'04) received the B.Sc. and M.Sc. degrees in computational mathematics from Shandong University, Shandong, China, in 1994 and 1997, respectively. He then received the M.Sc. degree in applied mathematics and the Ph.D. degree in mathematics and engineering from Queen's University, Kingston, ON, Canada, in 1998 and 2003, respectively.

From January to August 2003, he was a Postdoctoral Fellow in the Department of Mathematics and Statistics at Queen's University. He is currently a Postdoctoral Fellow in the Department of Electrical and Computer Engineering, University of Toronto, Toronto, ON, Canada. His current research interests include channel coding, iterative decoding, and information theory.

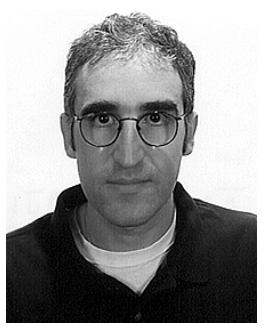

Fady Alajaji (S'90-M'94-SM'00) was born in Beirut, Lebanon, on May 1, 1966. He received the B.E. degree (with Distinction) from the American University of Beirut, Beirut, Lebanon, and the M.Sc. and Ph.D. degrees from the University of Maryland, College Park, in 1988, 1990, and 1994, respectively, all in electrical engineering.

He held a Postdoctoral appointment in 1994 at the Institute for Systems Research, University of Maryland. In 1995, he joined the Department of Mathematics and Statistics, Queen's University, Kingston, ON, Canada, where he is currently an Associate Professor of Mathematics and Engineering. Since 1997, he has also been cross-appointed to the Department of Electrical and Computer Engineering at the same university. He conducted research visits to the Department of Electrical and Computer Engineering, McGill University, Montreal, QC, Canada, in the fall of 2001, and to the Department of Mobile Communications, Technical University of Berlin, Berlin, Germany, in the winter of 2004. His research interests include information theory, joint source-channel coding, error-control coding, data compression, and digital communications.

Dr. Alajaji currently serves as Editor for Source and Source-Channel Coding for the IEEE TRANSACTIONS ON COMMUNICATIONS. He is Co-Chair of the Technical Program Committee of the 2004 Biennial Symposium on Communications. In 1999, he co-chaired and organized the 6th Canadian Workshop on Information Theory in Kingston, ON. He also served as Chair of the Selection Committee for the 2000 Canadian Award in Telecommunications. In 2001, he received the Premier's Research Excellence Award (PREA) from the Province of Ontario.

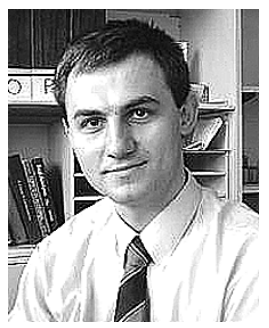

Jan Bajesy (S'94-M'00) received the B.Sc. degree in engineering from Harvard University, Cambridge, MA, in 1994, and the M.Sc. and Ph.D. degrees in electrical engineering from Princeton University, Princeton, NJ, in 1997 and 1999, respectively.

He is currently an Assistant Professor with the Department of Electrical and Computer Engineering, McGill University, Montreal, QC, Canada. His research focuses on channel coding, wireless communication systems, and optical signal processing.

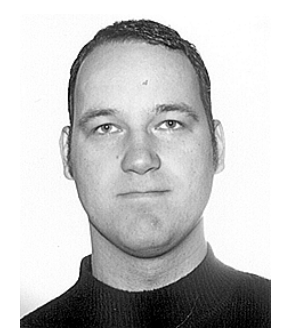

Patrick Mitran (S'01) received the Bachelor's and Master's degrees in electrical engineering, in 2001 and 2002, respectively, from McGill University, Montreal, QC, Canada. He is currently working toward the Ph.D. degree in the Division of Engineering and Applied Sciences, Harvard University, Cambridge, MA.

His research interests include iterative decoding theory, joint source-channel coding, detection, and estimation theory, as well as information theory. 